\title{
Entrenamiento en suturas para profesionales de la salud: experiencia inicial de un curso de formación continua
}

\author{
Suture training for health professionals: initial experience \\ of a continuing education course \\ Martín Inzunza,*,‡ Paloma Pozo, ${ }^{\S}$ Rodrigo Tejos, ${ }^{*, \|}$ Alfonso Navia,॥ \\ Cristián Jarry, *,‡ Pablo Achurra,` Julián Varas**
}

Palabras clave: Educación médica, profesionales de la salud, entrenamiento basado en simulación, suturas, retroalimentación.

Keywords: Medical education, health professionals, simulation-based training, sutures, feedback.

\section{* Fellow de Investigación, Centro de Simulación y \\ Cirugía Experimental. \\ ‡ Residente de Cirugía General, División de Cirugía, Facultad de Medicina. \\ $\S$ Interna de Medicina, Facultad de Medicina. \| Residente de Cirugía Plástica y Reconstructiva, División de Cirugía, Facultad de Medicina. ๑ Cirujano Digestivo, División de Cirugía, Facultad de Medicina. ** Coordinador Médico, Centro de Simulación y Cirugía Experimental. \\ Pontificia Universidad Católica de Chile.}

Recibido: 12/06/2020 Aceptado: 16/07/2020

doi: $10.35366 / 95229$

\section{RESUMEN}

Introducción: Se ha demostrado que la simulación es una alternativa efectiva para la enseñanza y el entrenamiento de procedimientos. Esta metodología implica la presencia permanente de tutores clínicos que entreguen retroalimentación efectiva, y se centra en estudiantes de medicina. Objetivos: Evaluar la adquisición de competencias en técnicas de sutura mediante un curso para profesionales de la salud, con una metodología mixta mediante videotutoriales y retroalimentación efectiva por expertos. Material y métodos: Se diseñó un programa con cuatro sesiones de entrenamiento presencial y práctica deliberada no presencial con videotutoriales. Se realizaron evaluaciones iniciales y finales en ciego para cuatro técnicas de sutura, aplicando escala OSATS y tiempo. Se fijó un puntaje de corte mínimo de aprobación de 20. Se compararon evaluaciones iniciales y finales, y se realizó análisis de subgrupo de acuerdo con la profesión, utilizando estadística no paramétrica. Resultados: El 100\% de los participantes logró un puntaje final $\geq 20$ en cada técnica, todos mejoraron de forma significativa sus habilidades basales y disminuyeron sus tiempos. El análisis de subgrupo no encontró diferencias estadísticamente significativas al finalizar el programa. Conclusión: El curso de suturas para profesionales de la salud con una metodología mixta de retroalimentación semipresencial resultó efectivo para la adquisición de las competencias propuestas.

\section{ABSTRACT}

Introduction: Simulation has been shown to be an effective alternative for teaching and training procedures. In the literature, the current methodology usually involves the permanent presence of clinical tutors for the delivery of effective feedback, also are focused on medical students. Objectives: To assess the acquisition of skills in suture techniques through a training program for health professionals, with a mixed methodology using video-tutorials and effective feedback by experts. Material and methods: A course was designed with four training sessions and deliberate home-practice with videotutorials between each session. Initial and final blind evaluations were performed for four specific suturing techniques, applying OSATS scale and execution Time. A cutoff score of 20 was set. Initial and final evaluations were compared, and subgroup analyzes were performed according to the profession, using non-parametric statistics. Results: $100 \%$ of the participants reached a final score $\geq 20$ in each technique, also all of them significantly improved their baseline skills and decreased their times. The subgroup analysis by profession did not find statistically significant differences at the end of the course. Conclusion: The suture training program for health professionals, with a mixed methodology through video-tutorials and feedback by experts, was effective for the acquisition of the proposed competencies.

\section{INTRODUCCIÓN}

L a sutura es una técnica quirúrgica básica utilizada por diversos profesionales de la salud como médicos, matronas, enfermeras, veterinarios, entre otros. Clásicamente, estos profesionales aprenden a suturar siendo alumnos durante la práctica directa con pacientes, bajo el modelo tradicional de enseñanza médica de Halsted: "mira uno, realiza uno, enseña uno". ${ }^{1}$
Una de las principales limitaciones de este modelo se observa en la enseñanza de procedimientos, debido a una falta de estandarización. ${ }^{2}$ Entre los factores que influyen en esto se advierte la desigual exposición clínica, la escasa disponibilidad de horario de docentes clínicos, la necesidad de instrumental especializado, la limitación de espacio físico y las características personales de docentes y alumnos. ${ }^{2,3}$ Por otra parte, la práctica directa en pacientes puede ge- 
nerar elevados niveles de estrés en alumnos que se enfrentan por primera vez al procedimiento; además, frecuentemente en pabellón se dispone de poco tiempo para retroalimentación y existe poco margen de error. 4,5

En este escenario, la simulación ha surgido como una herramienta complementaria ideal al permitir que los alumnos se expongan a diversas situaciones clínicas en un contexto seguro y protegido, previo a la práctica real. ${ }^{5}$ Un eje central de la educación basada en simulación es la retroalimentación. Ésta se define como el análisis que surge al comparar el rendimiento de un alumno con un estándar, y la entrega de dicha información al estudiante con el objetivo de mejorar su desempeño, ${ }^{6}$ lo cual lo estimula a reflexionar sobre sus habilidades y le otorga la posibilidad de generar un aprendizaje sólido y duradero. ${ }^{7,8}$

Existen estudios que han demostrado la efectividad del aprendizaje simulado de suturas; sin embargo, la mayoría se centra en alumnos de medicina o residentes de especialidades médicas. 2,4,9 Además, la metodología empleada habitualmente involucra la presencia permanente de tutores clínicos para la entrega de retroalimentación efectiva.

El objetivo de este estudio es evaluar la efectividad de un curso de formación continua para el entrenamiento simulado en técnicas de sutura para profesionales de la salud, que incluya una metodología mixta de retroalimentación con vi- deotutoriales estandarizados además de feedback presencial por docentes.

\section{MATERIAL Y MÉTODOS}

\section{Participantes}

Se invitó a participar a profesionales de la salud a un curso de educación continua. Los criterios de inclusión para participar fueron: edad $\geq 18$ años, acreditar título de profesional de la salud o ser estudiante regular de una carrera relacionada. Como criterio de exclusión se consideró la participación previa en algún programa formal de entrenamiento en suturas. Este estudio fue aprobado por el comité de ética institucional.

\section{Intervenciones}

Los alumnos fueron sometidos a un programa de entrenamiento de cuatro semanas de duración, con una metodología semipresencial. Para esto se entregó a cada alumno un kit de entrenamiento conformado por un pad de piel sintética, suturas e instrumental quirúrgico. Además, se entregaron videotutoriales sobre cuatro técnicas de sutura básica: punto simple, punto invertido, punto Donati y nudo cuadrado (con mano); mostrando aspectos específicos y estandarizados de su ejecución (Figura 1 A y B). Se solicitó a los participantes que entrenaran de forma deliberada en sus hogares,
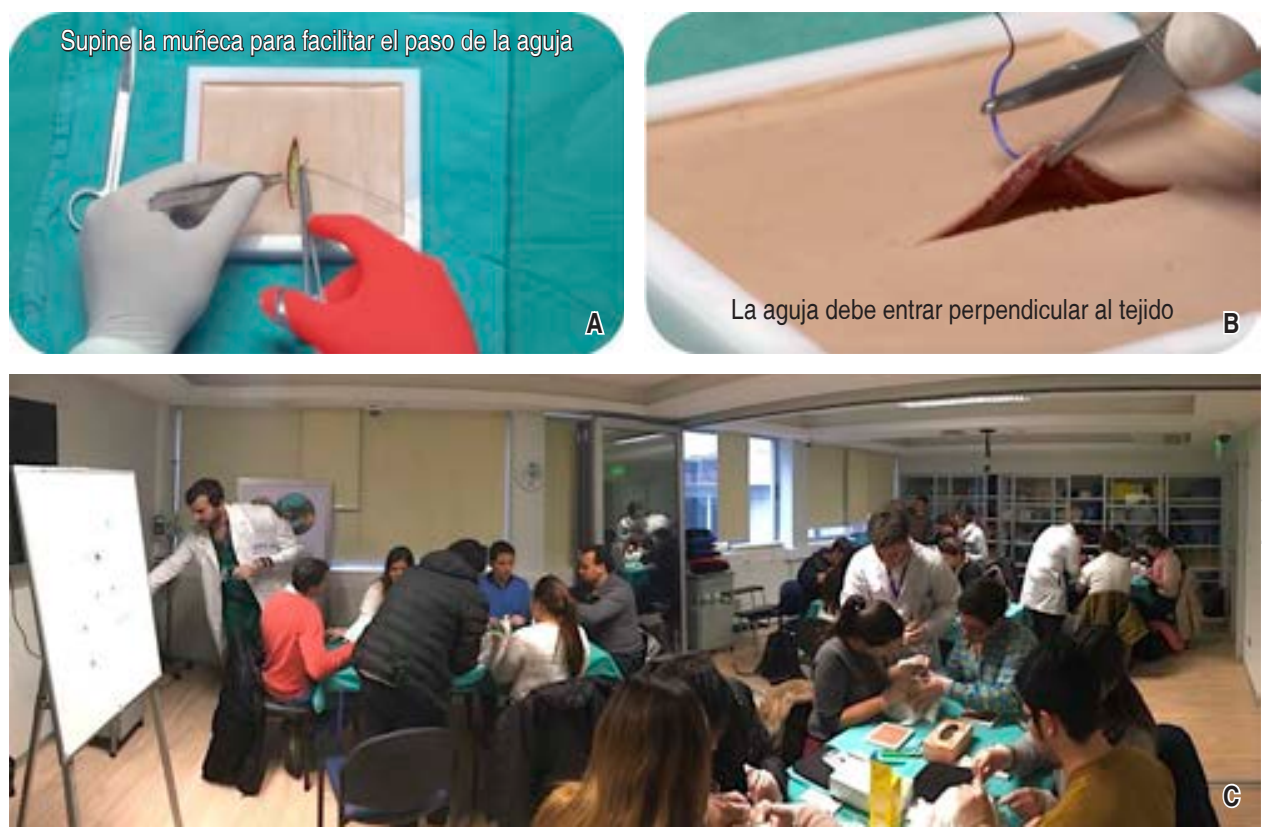

Figura 1:

A-B) Capturas de videotutorial mostrando y enfatizando aspectos especificos y estandarizados de la técnica de sutura. C) Módulo de entrenamiento. 
Muy frecuente

Frecuente

No sabe, no está seguro

Infrecuente

Muy infrecuente

Figura 2:

Encuesta en escala Likert: ¿Qué tan frecuente es la exposición a procedimientos

relacionados con el uso de suturas durante

su práctica clínica habitual? las diferentes técnicas de sutura. Durante la sesión de entrenamiento cada docente estuvo a cargo de un máximo de seis alumnos (Figura 1 C).

\section{Evaluación}

Previo al inicio del programa de entrenamiento se aplicó una encuesta a todos los participantes para determinar las características demográficas de la muestra. Además, se consultó sobre la frecuencia de exposición a procedimientos relacionados con el uso de suturas en su práctica clínica habitual, aplicando una escala Likert de cinco niveles (Figura 2).

Se realizaron dos evaluaciones formales del desempeño de los alumnos: una evaluación inicial y una evaluación final. Ambas evaluaciones fueron idénticas y consistieron en la videograbación de

Tabla 1: Características de la muestra. según disponibilidad horaria y necesidades de los participantes.

Se realizaron cuatro sesiones presenciales de entrenamiento de 120 minutos de duración cada una. Al inicio de cada sesión presencial los alumnos recibieron una clase expositiva con un académico sobre conceptos generales de cicatrización, tipo de instrumental y materiales quirúrgicos, manejo de heridas en distintos escenarios clínicos, manejo de heridas complejas y derivación. Cada alumno recibió retroalimentación presencial por docentes expertos sobre su desempeño al realizar

\begin{tabular}{lc}
\hline \multicolumn{2}{|c}{ Tabla 1: Características de la muestra. } \\
\hline Número de participantes & 28 \\
\hline Edad promedio años & 28.5 \\
Mujeres n (\%) & $14(50)$ \\
Profesiones (por categorías) n (\%) & \\
• Estudiantes de medicina & $2(7)$ \\
- Médicos generales & $14(50)$ \\
- Matronas & $6(21)$ \\
- Enfermeras & $3(11)$ \\
- Veterinarios & $3(11)$
\end{tabular}

Tabla 2: Análisis de subgrupo según profesión en escala Objective Structured Assessment of Technical Skill modificada, para la evaluación inicial y final.

\begin{tabular}{|c|c|c|c|c|c|c|c|}
\hline & & $\begin{array}{l}\text { Médicos } \\
(\mathrm{n}=14)\end{array}$ & $\begin{array}{l}\text { Estudiantes de } \\
\text { medicina } \\
(\mathrm{n}=2)\end{array}$ & $\begin{array}{l}\text { Matronas } \\
(\mathrm{n}=6)\end{array}$ & $\begin{array}{l}\text { Enfermeras } \\
\qquad(\mathrm{n}=3)\end{array}$ & $\begin{array}{l}\text { Veterinarios } \\
\qquad(\mathrm{n}=3)\end{array}$ & $\mathrm{p}$ \\
\hline \multirow[t]{2}{*}{ Punto simple } & Inicial & $16(13-18)$ & $18(16-20)$ & $14(12-15)$ & $15(13-16)$ & $15(14-16)$ & 0.229 \\
\hline & Final & $24(23-25)$ & $24(23-24)$ & $23(22-24)$ & $24(23-25)$ & $24(23-24)$ & 0.794 \\
\hline \multirow[t]{2}{*}{ Punto Invertido } & Inicial & $16(12-19)$ & $16(15-18)$ & $13(10-15)$ & $14(13-14)$ & $10(10-13)$ & 0.116 \\
\hline & Final & $22(20-25)$ & $21(20-21)$ & $22(20-23)$ & $25(23-25)$ & $21(21-22)$ & 0.465 \\
\hline \multirow[t]{2}{*}{ Punto Donati } & Inicial & $16(12-19)^{*}$ & $15(13-16)$ & $12(11-13)$ & $13(12-14)$ & $11(11-12)$ & 0.022 \\
\hline & Final & $22(20-24)$ & $22(21-22)$ & $20(20-21)$ & $23(22-23)$ & $21(21-22)$ & 0.581 \\
\hline \multirow[t]{2}{*}{ Nudo cuadrado } & Inicial & 15 (13-19) & $17(15-20)$ & $14(12-15)$ & $12(11-12)$ & $11(10-12)$ & 0.083 \\
\hline & Final & $24(22-25)$ & $24(24-25)$ & $22(20-25)$ & $25(25-25)$ & $21(21-23)$ & 0.279 \\
\hline
\end{tabular}

Mediana (RIQ). * = Diferencia estadísticamente significativa. 
Tabla 3: Análisis de subgrupo según profesión en tiempo de ejecución (minutos en decimales), para la evaluación inicial y final.

\begin{tabular}{|c|c|c|c|c|c|c|c|}
\hline & & $\begin{array}{l}\text { Médicos } \\
(n=14)\end{array}$ & $\begin{array}{l}\text { Estudiantes de } \\
\text { medicina } \\
(\mathrm{n}=2)\end{array}$ & $\begin{array}{l}\text { Matronas } \\
(\mathrm{n}=6)\end{array}$ & $\begin{array}{l}\text { Enfermeras } \\
\qquad(\mathrm{n}=3)\end{array}$ & $\begin{array}{l}\text { Veterinarios } \\
\quad(\mathrm{n}=3)\end{array}$ & $\mathrm{p}$ \\
\hline \multirow[t]{2}{*}{ Punto simple } & Inicial & $0.72 \pm 0.20$ & $0.82 \pm 0.12$ & $0.93 \pm 0.36$ & $0.61 \pm 0.25$ & $0.81 \pm 0.15$ & 0.362 \\
\hline & Final & $0.62 \pm 0.15$ & $0.55 \pm 0.03$ & $0.78 \pm 0.21$ & $0.59 \pm 0.08$ & $0.76 \pm 0.02$ & 0.117 \\
\hline \multirow[t]{2}{*}{ Punto invertido } & Inicial & $1.12 \pm 0.29$ & $1.46 \pm 0.37$ & $1.21 \pm 0.55$ & $1.39 \pm 0.83$ & $1.29 \pm 0.83$ & 0.817 \\
\hline & Final & $0.98 \pm 0.25$ & $0.61 \pm 0.18$ & $1.01 \pm 0.36$ & $0.78 \pm 0.13$ & $1.19 \pm 0.17$ & 0.471 \\
\hline \multirow[t]{2}{*}{ Punto Donati } & Inicial & $1.31 \pm 0.36$ & $1.68 \pm 0.33$ & $1.74 \pm 0.45$ & $1.73 \pm 0.92$ & $1.50 \pm 0.17$ & 0.285 \\
\hline & Final & $1.04 \pm 0.23$ & $0.63 \pm 0.15$ & $1.53 \pm 0.64$ & $1.01 \pm 0.16$ & $1.26 \pm 0.23$ & 0.362 \\
\hline \multirow[t]{2}{*}{ Nudo cuadrado } & Inicial & $0.63 \pm 0.22 *$ & $0.67 \pm 0.07$ & $0.94 \pm 0.19$ & $0.60 \pm 0.18$ & $1.07 \pm 0.24$ & 0.007 \\
\hline & Final & $0.59 \pm 0.20$ & $0.69 \pm 0.45$ & $0.51 \pm 0.19$ & $0.41 \pm 0.14$ & $0.69 \pm 0.03$ & 0.321 \\
\hline
\end{tabular}

cada participante ejecutando las cuatro técnicas de sutura básica descritas previamente: punto simple, punto invertido, punto Donati y nudo cuadrado. En cada videograbación se omitieron elementos que permitieran la identificación personal de los participantes. La evaluación inicial fue realizada el primer día del programa y previo a cualquier tipo de entrenamiento por los participantes. El objetivo de esta evaluación fue determinar el nivel de habilidades basales. La evaluación final fue realizada al término de la última sesión de entrenamiento presencial, al finalizar la cuarta semana del programa de entrenamiento. El objetivo de esta evaluación fue determinar el nivel de habilidades adquirido al finalizar el curso.

Dos evaluadores expertos que no participaron en ninguna actividad académica del programa, analizaron las videograbaciones de forma aleatoria y ciega a los participantes. Los evaluadores midieron los tiempos de ejecución y aplicaron una escala de evaluación global OSATS ${ }^{10}$ (Objective Structured Assessment of Technical Skill) modificada para asignar un puntaje de desempeño a cada técnica evaluada.

Se determinó un puntaje de corte mínimo de 20 puntos en la escala OSATS para considerar el logro de autonomía sobre cada técnica, siendo requisito para la aprobación del programa. Se consideró además un rango de tiempo máximo deseable de un minuto para punto simple y nudo cuadrado, y 1.5 minutos para punto invertido y Donati.

\section{Análisis estadístico}

El análisis estadístico se realizó mediante el programa SPSS Statistics ${ }^{\circledR}$ versión 22. Para el análisis de progresión del aprendizaje se utilizó la prueba de Wilcoxon para variables relacionadas. Para las comparaciones entre grupos se utilizó la prueba de Kruskal-Wallis. Un valor de $p<0.05$ se consideró estadísticamente significativo.

\section{RESULTADOS}

Un total de 30 profesionales de la salud se inscribieron para participar en el curso de formación continua, 28 de ellos aceptaron participar en el estudio y completaron todas las sesiones. La mediana de edad fue de 28.5 años, con 50\% de mujeres conformando la muestra. Cincuenta por ciento correspondieron a médicos generales, 11\% a enfermeras, 21\% a matronas, $7 \%$ a estudiantes de medicina y $11 \%$ a médicos veterinarios (Tabla 1). Todos los médicos generales, las matronas y los médicos veterinarios refirieron una exposición frecuente o muy frecuente a procedimientos relacionados con uso de suturas durante su práctica clínica habitual (Figura 2).

$\mathrm{Al}$ analizar la evaluación inicial de los participantes, ninguno superó el mínimo de suficiencia fijado en 20 puntos en la escala OSATS. Se observaron diferencias estadísticamente significativas en el análisis de subgrupo del punto Donati, donde el grupo de médicos generales obtuvo un puntaje OSATS mayor respecto al resto de los profesionales de la salud (mediana 16, RIQ 12-19; $p<0.05$ ) (Tabla 2). Lo mismo se observó al analizar el tiempo de ejecución en el nudo cuadrado, siendo menor en el grupo de médicos (media 0.63 segundos, DE $\pm 0.22 ; \mathrm{p}<0.05$ ), pero sin correlación con un puntaje OSATS suficiente (mediana 15, RIQ 13-19; $\mathrm{p}=\mathrm{NS}$ ) (Tabla 3). 

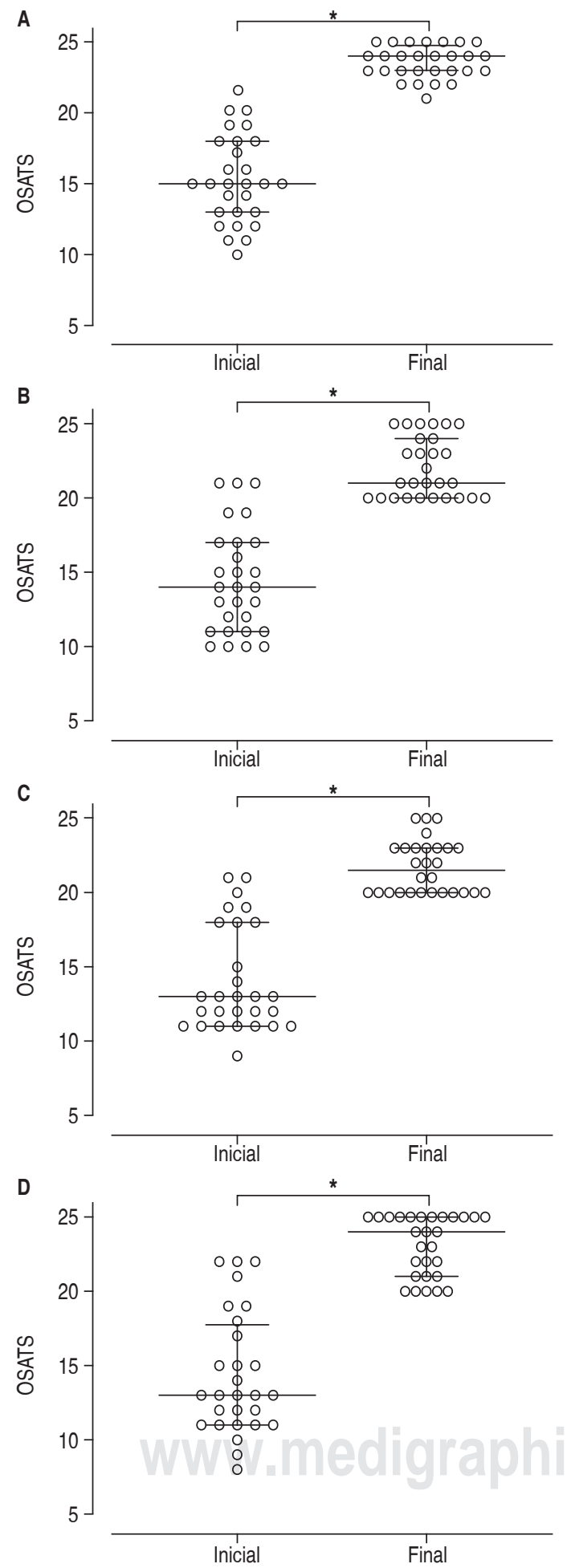

Figura 3: Comparación entre evaluación inicial y evaluación final en escala OSATS modificada. A) Punto simple. B) Punto invertido. C) Punto Donati. D) Nudo cuadrado.

$*=$ diferencia estadísticamente significativa.
Al término del programa de entrenamiento todos los participantes mejoraron sus habilidades basales para cada una de las técnicas enseñadas en función del puntaje OSATS, siendo esta diferencia estadísticamente significativa (Figura 3). De igual modo, todos los participantes disminuyeron sus tiempos en forma significativa para cada técnica (Figura 4).

Se realizó un análisis de subgrupo según profesión para el puntaje OSATS y tiempo de ejecución finales de cada una de las técnicas enseñadas, sin encontrar diferencias estadísticamente significativas (Tablas 2 y 3).

El $100 \%$ de los participantes obtuvo un puntaje OSATS final en cada técnica igual o mayor al puntaje mínimo de aprobación de 20 puntos, logrando autonomía en las cuatro técnicas enseñadas. Al evaluar el rango de tiempo de ejecución máximo deseable en cada técnica, dos participantes $(7 \%)$ excedieron este tiempo en el punto Donati, pero ambos con puntajes OSATS de 20 puntos, por lo que no afectó su aprobación (Figuras 3 y 4 ).

\section{DISCUSIÓN}

La educación médica ha evolucionado en los últimos años, incluyendo a la simulación como un método validado de enseñanza, ya que permite que los alumnos cometan errores de manera supervisada, pudiendo aprender de ellos antes de tener contacto con pacientes reales, en un ambiente seguro y estandarizado. De esta forma, la simulación logra disminuir las curvas de aprendizaje de procedimientos mediante la adquisición de competencias; además ha demostrado transferir estas competencias de forma efectiva a la práctica clínica. ${ }^{11-16}$

Este estudio demostró la adquisición de competencias en técnicas de sutura mediante un curso de formación continua con una metodología mixta de entrenamiento simulado y estandarizado para profesionales de la salud. Al analizar las habilidades basales de los participantes, se observa cierto grado de heterogeneidad. Esto se explica en parte, por una exposición relativamente más constante a suturas y procedimientos afines en un subgrupo de la muestra. Sin embargo, independiente de su profesión y experiencia previa, ninguno de los participantes logró niveles de suficiencia técnica aceptables en la evaluación inicial del curso, para ninguna de las técnicas analizadas. A su vez, al finalizar el curso el $100 \%$ de los participantes logró el mínimo de suficiencia 
técnica requerida para considerarlos autónomos en el procedimiento. Respecto al tiempo de ejecución máximo establecido, se observa que dos participantes se demoraron más de lo deseado en una sutura específica (punto Donati), pero sin repercutir en su calidad técnica. Por lo anterior, este hecho no influyó en su aprobación en el programa, priorizando una ejecución técnica satisfactoria por sobre el tiempo de ejecución.

Respecto a la retroalimentación durante el programa de entrenamiento, tanto el feedback realizado por los tutores como el obtenido de manera indirecta por los videotutoriales parecen lograr su objetivo de forma complementaria y costo-efectiva. En este contexto, al considerar las importantes limitaciones que puede tener un tutor clínico para lograr la enseñanza estandarizada de una habilidad técnica (horarios muy acotados para la docencia, tiempo prolongado de práctica, avance heterogéneo en el aprendizaje de los alumnos, entre otros), el complemento con videotutoriales estandarizados podría optimizar el tiempo exclusivo de retroalimentación presencial por los docentes, además de favorecer y estimular la práctica personal del alumno. Nuevas metodologías y herramientas de retroalimentación no presenciales se encuentran en desarrollo para el entrenamiento de cirujanos a distancia, ${ }^{3}$ las cuales en el futuro podrían ser aplicadas a la enseñanza de técnicas de sutura básica.

Un aspecto relevante a destacar es la falta de enseñanza estandarizada en nuestro país respecto a un procedimiento tan habitual en la práctica clínica como lo es la sutura. ${ }^{2,9}$ En Chile, tanto médicos como matronas, enfermeras y veterinarios son profesionales que pueden enfrentarse a esto en su práctica habitual. Considerando los avances en el proceso de enseñanza-aprendizaje en el área de la salud, el resguardo de la seguridad del paciente, y el fuerte desarrollo de la simulación clínica, ${ }^{5}$ parece necesario incentivar aún más su utilidad para la adquisición de competencias. Asimismo, es importante favorecer y extender su aplicación en las mallas curriculares de las distintas carreras de la salud y afines, con evidencia fundamentada de su utilidad. 2,9,17

Existe poca evidencia sobre el uso de la simulación para la práctica de suturas en otras carreras del área de la salud distintas a medicina. Un estudio realizado por Guler y colegas en estudiantes de obstetricia comparó la ejecución de la episiotomía en un modelo de lengua de vaca versus otro de material sintético. Posterior al entre-
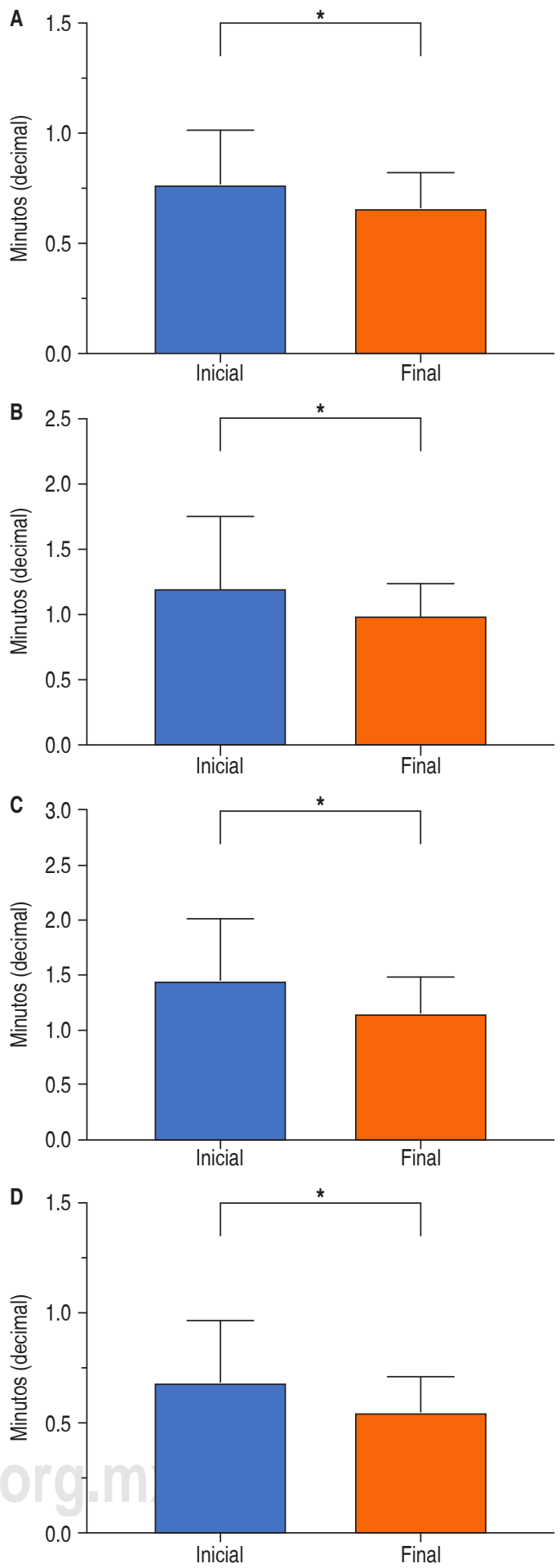

Figura 4: Comparación entre evaluación inicial y evaluación final en función del tiempo de ejecución (minutos en decimales). A) Punto simple. B) Punto invertido. C) Punto Donati. D)

Nudo cuadrado.

* = diferencia estadísticamente significativa. 
namiento, los estudiantes realizaron la técnica en pacientes reales. Se midió mediante una encuesta si se sintieron mejor preparados tras la práctica en simulación. Los resultados muestran un aumento en la confianza que sienten los estudiantes al momento de realizar el procedimiento en ambos modelos, sin diferencias estadísticamente significativas. ${ }^{18}$ Otro estudio similar, también en estudiantes de obstetricia, describe una mejoría en la percepción de la técnica de episiotomía al entrenarse en un modelo de lengua de buey. ${ }^{19}$ Una publicación australiana compara el desempeño de enfermeras a nivel terciario al suturar heridas cutáneas previo y posterior a realizar un curso estructurado de simulación. Los resultados se midieron con base en una encuesta aplicada a las participantes. Se observó una mejoría estadísticamente significativa en la percepción de sus habilidades procedurales. ${ }^{20}$ En suma, a través de estos estudios se desprende que los alumnos parecen sentirse mejor preparados para realizar procedimientos en pacientes luego de un entrenamiento previo mediante simulación, lo cual genera la necesidad de ampliar y extender su uso como herramienta docente regular.

Nuestro estudio propone una metodología de entrenamiento estructurada, estandarizada y costo-efectiva para profesionales de diversas áreas de la salud. El uso de videotutoriales optimiza el tiempo docente presencial, lo cual facilita la reproducibilidad de esta metodología. Además, la duración acotada de sólo cuatro sesiones presenciales también podría facilitar su implementación. Si bien, el público objetivo de este programa fueron profesionales de la salud ya egresados, ofreciendo una alternativa a quienes recibieron una metodología de enseñanza tradicional no estandarizada, enfatizamos la factibilidad y la necesidad de extenderlo a las diversas mallas curriculares de estas carreras de la salud.

Una de las limitaciones de este estudio es que no se cuantificó el tiempo destinado al entrenamiento personal, ni el número de veces que los alumnos consultaban los videotutoriales. Estas variables podrían explicar en cierta medida las diferencias específicas o la curva de aprendizaje propia de cada alumno, pero también podrían ser fuente de sesgo al depender de registros personales de los participantes.

\section{CONCLUSIÓN}

El curso de formación continua orientado a profesionales de la salud, con una metodología mixta semipresencial, resultó efectivo en la adquisición de las competencias propuestas. El aprendizaje mediante videotutoriales de libre consulta, junto con retroalimentación efectiva por docentes expertos durante sesiones de entrenamiento, parece ser una alternativa válida, segura y costo-efectiva para el entrenamiento de técnicas de suturas en profesionales de la salud, optimizando y estandarizando su enseñanza-aprendizaje.

\section{REFERENCIAS}

1. Tejos R, Avila R, Inzunza M, Achurra P, Castillo R, Rosberg A, et al. Impact of a simulated training program in a three- year general surgery residency. $A B C D$ Arq Bras Cir Dig. 2019; 32 (2): 1-5.

2. González LR, Molina ZH, García-Huidobro DM, Stevens MP, Jadue TA, Riquelme UA, et al. Implementación de taller de monitores de sutura en alumnos de pregrado de medicina. Rev Cir. 2019; 71 (2): 122-128.

3. Quezada J, Achurra P, Jarry C, Asbun D, Tejos R, Inzunza $M$, et al. Minimally invasive tele-mentoring opportunity-the MITO project. Surg Endosc. 2020; 34 (6): 2585-2592.

4. Zundel S, Meder A, Zipfel S, Herrmann-Werner A. The surgical experience of current non-surgeons gained at medical school: a survey analysis with implications for teaching today's students. BMC Med Educ. 2015; 15 (1): $1-7$

5. Jarry C, Inzunza M, Quezada J, Marino C, Zamorano E, Alvarado V, et al. Gimnasio de simulación quirúrgica: una herramienta educacional factible de entrenamiento continuo. Experiencia de un centro universitario. Simulación Clínica. 2019; 1: 18-24.

6. Van De Ridder JMM, Stokking KM, McGaghie WC, Ten Cate OTJ. What is feedback in clinical education? Med Educ. 2008; 42 (2): 189-197.

7. Burns CL. Using debriefing and feedback in simulation to improve participant performance: an educator's perspective. Int J Med Educ. 2015; 6: 118-120.

8. Gaunt A, Patel A, Rusius V, Royle TJ, Markham DH, Pawlikowska T. 'Playing the game': How do surgical trainees seek feedback using workplace-based assessment? Med Educ. 2017; 51 (9): 953-962.

9. Alvarado J, Henríquez JP, Castillo R, Sosa J, León F, Varas J, et al. Programa pionero de simulación en sutura para estudiantes de medicina de pregrado. Rev Cir. 2015; 67: 480-485.

10. Martin JA, Regehr G, Reznick R, Macrae H, Murnaghan J, Hutchison C, et al. Objective structured assessment of technical skill (OSATS) for surgical residents. $\mathrm{Br}$ J Surg. 1997; 84 (2): 273-278.

11. Vassiliou MC, Dunkin BJ, Marks JM, Fried GM. FLS and FES: comprehensive models of training and assessment. Surg Clin North Am. 2010; 90 (3): 535-558.

12. Tavakol M, Mohagheghi MA, Dennick R. Assessing the skills of surgical residents using simulation. J Surg Educ. 2008; 65 (2): 77-83.

13. Grantcharov TP, Funch-Jensen P. Can everyone achieve proficiency with the laparoscopic technique? Learning curve patterns in technical skills acquisition. Am J Surg. 2009; 197 (4): 447-449. 
14. Orzech N, Palter VN, Reznick RK, Aggarwal R, Grantcharov TP. A comparison of 2 ex vivo training curricula for advanced laparoscopic skills: a randomized controlled trial. Ann Surg. 2012; 255 (5): 833-839.

15. Bansal VK, Raveendran R, Misra MC, Bhattacharjee H, Rajan K, Krishna A, et al. A prospective randomized controlled blinded study to evaluate the effect of short-term focused training program in laparoscopy on operating room performance of surgery residents (CTRI /2012/11/003113). J Surg Educ. 2014; 71 (1): 52-60.

16. Varas J, Mejía R, Riquelme A, Maluenda F, Buckel E, Salinas J, et al. Significant transfer of surgical skills obtained with an advanced laparoscopic training program to a laparoscopic jejunojejunostomy in a live porcine model: feasibility of learning advanced laparoscopy in a general surgery residency. Surg Endosc Other Interv Tech. 2012; 26 (12): 3486-3494.

17. Davis CR, Toll EC, Bates AS, Cole MD, Smith FCT Surgical and procedural skills training at medical school - a national review. Int J Surg. 2014; 12 (8): 877-882.
18. Guler H, Cetin P, Yurtsal ZB, Cesur B, Bekar M, $U$ çar $T$, et al. Effect of episiotomy training with beef tongue and sponge simulators on the self-confidence building of midwifery students. Nurse Educ Pract. 2018; 30: 1-6.

19. Selo-Ojeme D, Ojutiku D, Ikomi A. Impact of a structured, hands-on, surgical skills training program for midwives performing perineal repair. Int J Gynecol Obstet. 2009; 106 (3): 239-241.

20. Middleton R. Suturing as an advanced skill for registered nurses in the emergency department. Aust J Rural Health. 2006; 14 (6): 258-262.
Correspondencia:
Dr. Julián Varas C
Marcoleta Núm. 377,
Santiago de Chile.
Tel. +56223546483
E-mail: jevaras@uc.cl 